\title{
The effect of combined treatment with sodium phenylbutyrate and cisplatin, erlotinib, or gefitinib on resistant NSCLC cells
}

This article was published in the following Dove Press journal:

Clinical Pharmacology:Advances and Applications

\author{
Maha S Al-Keilani' \\ Karem H Alzoubi ${ }^{1}$ \\ Saied A Jaradat ${ }^{2,3}$ \\ 'Department of Clinical Pharmacy, \\ College of Pharmacy, Jordan \\ University of Science and Technology, \\ Irbid 22। I0, Jordan; ${ }^{2}$ Department \\ of Biotechnology and Genetic \\ Engineering, Jordan University of \\ Science and Technology, Irbid 22I I0, \\ Jordan; ${ }^{3}$ Princess Haya Center for \\ Biotechnology, Jordan University of \\ Science and Technology, Irbid 22I I0, \\ Jordan
}

Background: Chemotherapy resistance is the main cause of the marginal clinical benefit of platinum-based chemotherapy and tyrosine kinase inhibitors in advanced non-small-cell lung cancer (NSCLC). Thus, the identification of new therapeutic agents that can enhance the sensitivity of these drugs is of clinical importance. Histone deacetylase inhibitors (HDACIs) are emerging as new promising agents with strong antiproliferative effects against different types of cancers. This study investigates the synergistic potential of sodium phenylbutyrate (NaPB) added on top of standard chemotherapy used against NSCLC.

Objective: The objective of this study was to evaluate the ability of NaPB to overcome the resistance of NSCLC cell lines to cisplatin, gefitinib, and erlotinib.

Methods: MTT cell proliferation assay was used to measure the anticancer effects of cisplatin, erlotinib, or gefitinib alone or combined with various concentrations of NaPB against A549, Calu1, and H1650 NSCLC cell lines. Synergism was estimated by measuring synergy value (R), which is equal to the ratio of $\mathrm{IC}_{50}$ of each primary drug alone divided by combination $\mathrm{IC}_{50} \mathrm{~s}$. Student's $t$-test analysis was used to evaluate the potential differences between $\mathrm{IC}_{50}$ values. ANOVA followed by Tukey's post hoc was used to evaluate the potential differences among monotherapy and combination treatment groups. Analyses were performed using R 3.3.2 software. $P$-value $<0.05$ was considered to be statistically significant.

Results: NaPB was shown to inhibit the growth of A549, Calu1, and H1650 cell lines in a dose-dependent manner ( $\mathrm{IC}_{50} 10,8.53$, and $4.53 \mathrm{mM}$, respectively). Furthermore, the addition of $\mathrm{NaPB}$ along with cisplatin, erlotinib, or gefitinib to A549, Calu1, and H1650 cell lines resulted in a synergistic antiproliferative effect against the three NSCLC cell lines $(R>1.6, P$-value $<0.05$ ), thus suggesting that $\mathrm{NaPB}$ can potentiate the effect of cisplatin, erlotinib, and gefitinib on A549, Calu1, and H1650 cell lines.

Conclusion: Current results suggest a potential role of $\mathrm{NaPB}$ as a sensitizing agent in NSCLC. Keywords: NSCLC, resistance, histone deacetylase inhibitor, sodium phenylbutyrate, synergism, sensitizing chemotherapy

\section{Introduction}

Lung cancer is a health concern worldwide since it is one of the most common cancers accounting for $17 \%$ of cancer cases in men and $7 \%$ of cases in women. It is also the leading cause of cancer-related mortality in both sexes being responsible for about $19 \%$ of deaths. ${ }^{1}$ The non-small-cell lung cell (NSCLC) accounts for the majority of lung cancer cases ${ }^{2}$ however, about three quarters of the patients with NSCLC are diagnosed at late stages (IIIB and IV). ${ }^{3}$ Platinum-based chemotherapy is considered the main-
Correspondence: Maha S Al-Keilan Department of Clinical Pharmacy, College of Pharmacy, Jordan University of Science and Technology, PO Box 3030, Irbid 22110 , Jordan

Tel +96227201000

Email mskeilani@just.edu.jo 
stay of therapy for patients with advanced, metastatic, and recurrent NSCLC. ${ }^{4,5}$ The marginal clinical benefit associated with the use of platinum-based chemotherapy and targeted molecular therapy including the epidermal growth factor receptor tyrosine kinase inhibitors (EGFR-TKIs), gefitinib and erlotinib, in advanced NSCLC is mainly due to resistance, either intrinsic or acquired. ${ }^{4-6}$ Thus, identification of new therapeutic agents that can be used as sensitizing agents for the treatment of NSCLC is unequivocal.

Histone deacetylase inhibitors (HDACIs) are under investigation as promising future chemotherapeutic agents for the treatment of various types of cancers. Examples are vorinostat, romidepsin, panobinostat, and belinostat that have been approved by the US Food and Drug Administration for the treatment of hematological cancers. ${ }^{7,8}$

The HDACIs showed anticancer effects through the inhibition of tumor-cell growth, induction of cellular apoptosis, and differentiation in malignant cells. ${ }^{9}$ Their recruitment in the anticancer regimen enhanced the cytotoxicity of several anticancer agents. ${ }^{10,11}$ Sodium phenylbutyrate (NaPB), the salt of a short-chain fatty acid, is another HDACI and was the first of its class to be encountered. ${ }^{12,13}$ Several studies revealed the antineoplastic potential of this agent in different types of cancers including lung cancer using both in vitro and in vivo studies. ${ }^{14-17}$ Unfortunately, in vivo studies and clinical trials failed to reveal beneficial anticancer effect of $\mathrm{NaPB}$ when used as monotherapy and suggested that it is better for $\mathrm{NaPB}$ to be used as part of combination chmotherapies. ${ }^{18}$ To the best of our knowledge, there are no in vitro studies that focus on the synergistic potential of NaPB in combination with standard chemotherapy for the treatment of NSCLC. Thus, in this study, the ability of NaPB to sensitize the NSCLC cell lines to cisplatin, erlotinib, and gefitinib and overcome their resistance against these agents was investigated.

\section{Materials and methods}

\section{Cell culture and drug treatment}

Human NSCLC, A549 (Kirsten Rat Sarcoma Virus [KRAS]mutant; G12S, EGFR-wild-type), Calu1 (KRAS-mutant; G12C, EGFR-wild-type), and H1650 (KRAS-wild-type, EGFR-mutant; a deletion in exon 19 of the EGFR gene delE746A750) cell lines were purchased from the American Type Culture Collection (ATCC, Manassas, VA, USA). The A549 cell line was cultured in DMEM (Euroclone S.P.A, Pero, MI, Italy), and Calu1 and H1650 cell lines were cultured in Roswell Park Memorial Institute medium (RPMI; Euroclone). Culture media were supplemented with $10 \%$ fetal bovine serum (Euroclone) and 1\% penicillin/streptomycin
(100 IU/mL penicillin and $100 \mu \mathrm{g} / \mathrm{mL}$ streptomycin; Euroclone). Cell lines were maintained in a humidified incubator of $95 \%$ air and $5 \% \mathrm{CO}_{2}$ atmosphere at $37^{\circ} \mathrm{C}$.

Cisplatin, erlotinib, gefitinib, and NaPB were purchased from Combi-Blocks (San Diego, CA, USA). Cisplatin, erlotinib, and gefitinib were dissolved in dimethyl sulfoxide (DMSO) at concentrations of 100, 10, and $10 \mathrm{mM}$, respectively. NaPB was dissolved in nuclease-free water at a concentration of $2 \mathrm{M}$. All drug stocks were stored in dark-colored bottles at $-20^{\circ} \mathrm{C}$ as stock solutions. The drugs were diluted with culture media to the required concentrations immediately before use. Before treatment with the drugs, the medium was removed when cells were adherent and about $80 \%$ confluent.

\section{MTT assay}

The cells were seeded in 96-well plates at a density of $5 \times 10^{3}$ in $200 \mu \mathrm{L}$ of medium per well, and the cells were incubated and allowed to attach overnight. After that media were replaced with fresh ones containing various concentrations of drugs; NaPB (0-32 mM), cisplatin (0-96 $\mu \mathrm{M})$, erlotinib $(0-32 \mu \mathrm{M})$, or gefitinib $(0-32 \mu \mathrm{M})$ alone or in combination with three different concentrations of $\mathrm{NaPB}\left(\mathrm{IC}_{50}, 0.5 \times \mathrm{IC}_{50}\right.$, $2 \times \mathrm{IC}_{50}$ ). Cells grown in medium alone (for treatment with $\mathrm{NaPB}$ only) or containing an equivalent amount of DMSO (for other treatment conditions) served as control.

After incubation for 72 hours at $37^{\circ} \mathrm{C}, 20 \mu \mathrm{L}$ of MTT dye was added to the treated cells $(5 \mathrm{mg} / \mathrm{mL}$ in PBS) and then incubated for 3 hours at $37^{\circ} \mathrm{C}$. After that, the MTT dye was discarded, and the formazan product was dissolved by adding $100 \mu \mathrm{L}$ of DMSO to each well, followed by shaking for 5 minutes. The spectrophotometric absorbance of the plates was measured using an ELISA plate reader at 570 $\mathrm{nm}$ with a reference wavelength of $690 \mathrm{~nm}$. Cell viability was calculated as follows: absorbance of the experimental group/absorbance of the control group. The $\mathrm{IC}_{50}$ value was defined as the concentration that inhibited cell proliferation by $50 \%$ when compared to that of the controls. $\mathrm{IC}_{50}$ values were calculated using Compusyn software 1.0 (Combosyn Inc., Paramus, NJ, USA). All measurements were done in triplicates, and the values shown in the figure and tables represent the mean value of the three wells.

\section{Synergism analyses}

The three NSCLC cell lines were treated with cisplatin, erlotinib, or gefitinib alone or in combination with $\mathrm{NaPB}$ at fixed concentrations that corresponded to the $\mathrm{IC}_{50}, 0.5 \times \mathrm{IC}_{50}$, and $2 \times \mathrm{IC}_{50}$ in each cell line. The degree of interaction between $\mathrm{NaPB}$ and cisplatin, erlotinib, or gefitinib was calculated 
through the synergy values $(\mathrm{R})$ as previously described by Martinez-Marignac et al. ${ }^{19}$ The $\mathrm{R}$ was measured by dividing the value of $\mathrm{IC}_{50}$ of each primary drug alone (cisplatin, erlotinib, or gefitinib) divided by $\mathrm{IC}_{50}$ value for the combination therapy. $\mathrm{R}$ values $>1.6,=1$, and $<1$ indicated synergism, additive effect, and antagonism, respectively.

\section{Statistical analyses}

Student's $t$-test analysis was used to evaluate the potential differences between $\mathrm{IC}_{50}$ values. ANOVA followed by Tukey's post hoc test was used to evaluate the potential differences among monotherapy and combination treatment groups. Analyses were performed using R 3.3.2 software. $P$-value $<0.05$ was considered to be statistically significant.

\section{Results}

\section{NaPB inhibited the growth of NSCLC cells}

Three NSCLC cell lines A549, Calu1, and H1650 were utilized. Initially, the effects of NaPB on the proliferation of the three cell lines were investigated, using the MTT assay performed after 72 hours of treatment. The NaPB inhibited the growth of the three cell lines in a dose-dependent manner. The $\mathrm{IC}_{50}$ values were $10,8.5$, and $4.5 \mathrm{mM}$ in A549, Calu1, and $\mathrm{H} 1650$, respectively (Table 1).

\section{Synergistic growth inhibition by NaPB in combination with cisplatin, erlotinib, and gefitinib}

For the combined treatment, the three NSCLC cell lines were treated with a series of concentrations of cisplatin $(0-96 \mu \mathrm{m})$, erlotinib $(0-32 \mu \mathrm{M})$, or gefitinib $(0-32 \mu \mathrm{M})$ in the presence or absence of three concentrations of $\mathrm{NaPB}$ that corresponded to $0.5 \times \mathrm{IC}_{50}, \mathrm{IC}_{50}$, and $2 \times \mathrm{IC}_{50}$.

A549 and Calu1 cell lines containing activating KRAS mutations causing resistance to gefitinib and erlotinib were exposed to 5, 10, and $20 \mathrm{mM}$ and 4, 8, and $16 \mathrm{mM}$ of $\mathrm{NaPB}$, respectively. While $\mathrm{H} 1650$ cell lines containing an activating

Table I IC ${ }_{50}$ values

\begin{tabular}{|l|l|l|l|}
\hline & \multicolumn{3}{|l|}{ NSCLC cell line } \\
\cline { 2 - 4 } & A549 & Calu I & HI650 \\
\hline NaPB $(\mathrm{mM})$ & 9.94 & 8.53 & 4.53 \\
Cisplatin $(\mu \mathrm{M})$ & 416.43 & 240.89 & 628.38 \\
Erlotinib $(\mu \mathrm{M})$ & 15.86 & 41.36 & 3.18 \\
Gefitinib $(\mu \mathrm{M})$ & 6 & 11.93 & 8.79 \\
\hline
\end{tabular}

Note: $I_{50}$ is the inhibitory concentration of $50 \%$ viability.

Abbreviations: NaPB, sodium phenylbutyrate; NSCLC, non-small-cell lung cancer.
EGFR deletion and are resistant to erlotinib and gefitinib were exposed to 2, 4, and $8 \mathrm{mM}$ of $\mathrm{NaPB}$. The growth inhibitory effect was measured in the three cell lines by MTT assays. As shown in Figure 1, current results revealed that NaPB enhanced the anticancer effects of the three tested chemotherapeutic agents in a dose-dependent manner. Moreover, the addition of NaPB to A549, Calu1, and H1650 along with cisplatin, erlotinib, or gefitinib resulted in synergistic antiproliferative effect against the three NSCLC cell lines (Table $2 ; R>1.6, P$-value $<0.05$ ), thus suggesting that $\mathrm{NaPB}$ can potentiate the effect of cisplatin, erlotinib, and gefitinib on A549, Calu1, and H1650. The degree of synergism was the highest in A549 cell line.

\section{Discussion}

Platinum-based regimens and EGFR-TKIs remain the major chemotherapy for NSCLC, but resistance to these agents has negatively affected the overall survival of patients. Resistance to anticancer therapy can occur through different mechanisms such as impaired permeability, DNA mutations, metabolic changes, DNA damage repair, and epigenetic alterations. ${ }^{11}$ Epigenetic mechanisms are critical for normal genetic development of the mammalian cells, and cancer is believed to develop due to the accumulation of genetic and epigenetic aberrations through a multistep process, ${ }^{10}$ creating a heterogeneous disease at both cellular and molecular levels. ${ }^{20}$ Thus, epigenetic aberrations play a major role in cancer development and progression and anticancer therapy resistance. ${ }^{11}$

There is a continued interest in the inclusion of relatively safe drugs such as HDACIs to the conventional chemotherapy. There are also several lines of evidence that support the effectiveness of HDACIs in enhancing the anticancer activity of cisplatin and TKIs. In ovarian cancer cell lines, overexpression of HDAC isoforms resulted in resistance to cisplatin, ${ }^{21}$ and combination of cisplatin with panobinostat, a HDACI, enhanced ovarian cells sensitivity. ${ }^{22}$ Synergistic anticancer effect was also evident when cisplatin was combined with belinostat in NSCLC cells. ${ }^{22,23}$ In patients with NSCLC, HDAC class I and II overexpression served as a negative prognostic biomarker. ${ }^{24,25}$ On the other hand, combining trichostatin A with erlotinib was associated with the stimulation of NSCLC cell apoptosis. ${ }^{26}$

The current study was performed in an attempt to identify the synergistic potential of the HDACI NaPB when combined with the conventional chemotherapy in NSCLC utilizing cell lines that have genetic mutations making them resistant to therapy. KRAS and EGFR are two oncogenes that are among the genes that are frequently mutated in lung cancer 
A
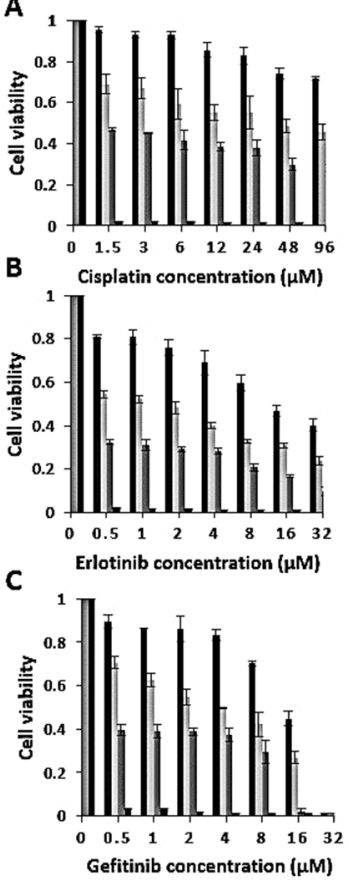

- Cisplatin

a Cisplatin $+5 \mathrm{mM}$ NaPB

- Cisplat in + $20 \mathrm{mM}$ NaPB

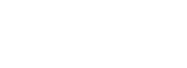

Erlotinib
aErlotinib +5 mM NaPB
Erlotinib + $10 \mathrm{mM}$ NaPB Erlotinib + $10 \mathrm{mM}$ NaPB
Erlotinib + $20 \mathrm{mM}$ NaPB$$
\text { (2) }
$$

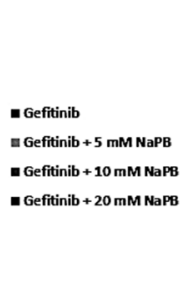

D

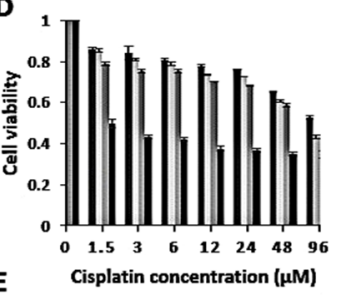

E 1
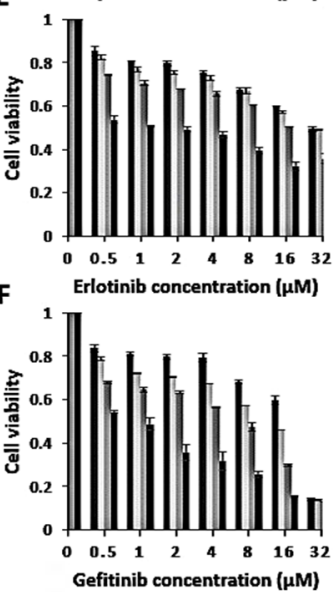

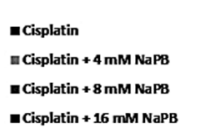

G

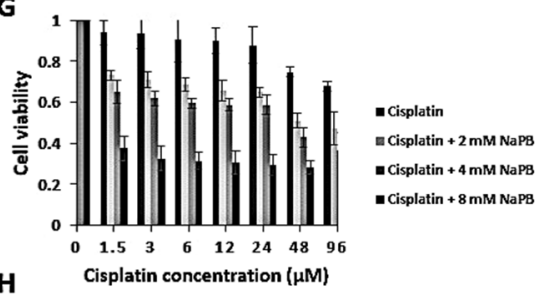

H

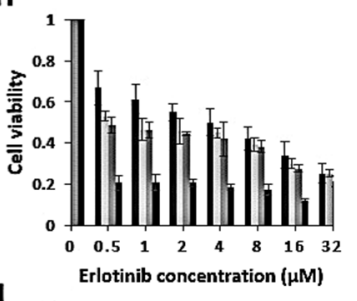

- Erlotinit

Erlotinib +2 mM NaPB

- Erlotinib $+4 \mathrm{mM} \mathrm{NaPB}$

- Erlotinib + 8 mM NaPB

I

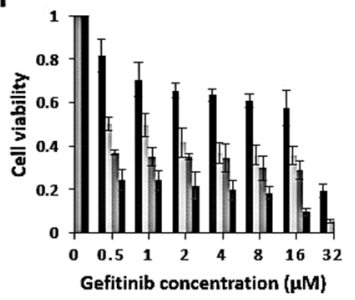

- Gefitinib

efitinib $+2 \mathrm{mM} \mathrm{NaPB}$

Gefitinib $+4 \mathrm{mM}$ NaPB

- Gefitinib + 8 mm NaPB

Figure I NaPB combined with cisplatin, erlotinib, or gefitinib showed potential synergism in A549 (A-C), Calul (D-F), and HI650 (G-I) NSCLC cell lines.

Notes: Cell lines were incubated for 72 hours with a series of concentrations of cisplatin, erlotinib, or gefitinib alone or in combination with NaPB at three fixed concentrations corresponding to $0.5 \times I C_{50}, I C_{50}, 2 \times I C_{50}$ in each cell line. Cells grown in medium containing an equivalent amount of DMSO served as control. After treatment, cell proliferation was measured using MTT assay. Data are expressed as mean \pm SD of triplicates. ANOVA followed by Tukey's post hoc test was used to evaluate the potential differences among monotherapy and combination treatment groups. $P$-value $<0.05$ was considered to be statistically significant.

Abbreviations: DMSO, dimethyl sulfoxide; NaPB, sodium phenylbutyrate; NSCLC, non-small-cell lung cancer.

resulting in treatment resistance mainly to the EGFR-TKIs erlotinib and gefitinib. ${ }^{27,28}$ Studies on chemotherapy-resistant NSCLC cell lines have shown a synergistic potential of the HDACIs, panobinostat and romidepsin when combined with erlotinib, and vorinostat when combined with gefitinib and erlotinib. ${ }^{29-31}$ Thus, HDACIs hold a big promise in overcoming resistance to erlotinib and gefitinib in NSCLC cell lines harboring mutations in KRAS or EGFR genes.

$\mathrm{NaPB}$ was more effective in inhibiting H1650 cell proliferation than Calu1, consistent with the results of the previous studies that showed a cell-type-specific anticancer effect of $\mathrm{NaPB} .{ }^{18}$ Current results also revealed that the resistance of NSCLC cells to cisplatin, erlotinib, and gefitinib can be overcome via combination of these agents with NaPB. Moreover, the current study has demonstrated that NaPB inhibited the growth of NSCLC cell lines in a dose-dependent manner and that it synergized with the three drugs in inhibition of tumor cell growth as evidenced by the synergy values $(\mathrm{R})$.

This synergistic effect can be explained by several mechanisms, as NaPB inhibits class I and IIa HDAC enzymes resulting in reprogramming of gene expression and posttranslational modifications. ${ }^{18,32} \mathrm{~A}$ gene expression microarray analysis revealed that sodium butyrate, a derivative of $\mathrm{NaPB}$, downregulated 100 cell cycle-associated genes in NSCLC cell lines. ${ }^{33}$ As a consequence, NaPB therapy is associated with the inhibition of cellular proliferation, metastasis, and angiogenesis, induction of cell cycle arrest and apoptosis.

\section{Conclusion}

Current results indicate that the combination of $\mathrm{NaPB}$ with the selected anticancer agents in NSCLC is a successful strategy that may reduce the risk of resistance. This supports the use of NaPB as sensitizing chemotherapy in NSCLC rather than a monotherapy. Future work is suggested to focus on testing the tolerability and efficacy of such combinations in preclinical models.

\section{Acknowledgment}

This study received grant support from Deanship of Scientific Research at Jordan University of Science and Technology (20150158). 


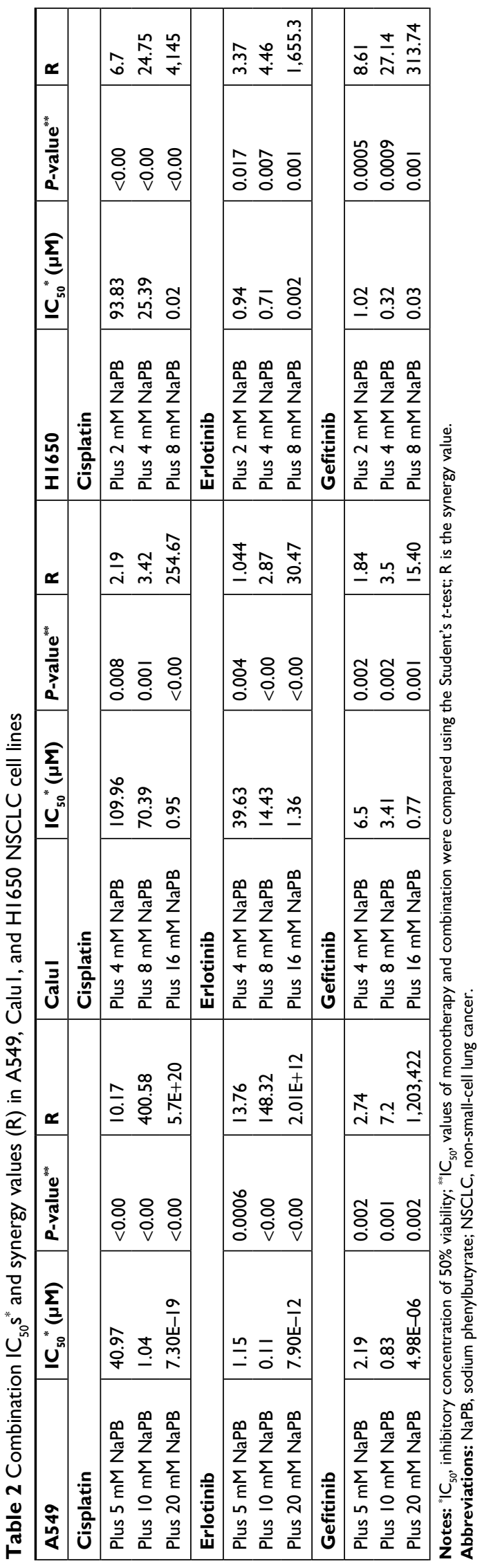

\section{Author contributions}

All authors contributed toward data analysis, drafting, and critically revising the paper and agree to be accountable for all aspects of the work.

\section{Disclosure}

The authors report no conflicts of interest in this work.

\section{References}

1. Cheng TY, Cramb SM, Baade PD, Youlden DR, Nwogu C, Reid ME. The international epidemiology of lung cancer: Latest trends, disparities, and tumor characteristics. J Thorac Oncol. 2016;11(10):1653-1671.

2. Molina JR, Yang P, Cassivi SD, Schild SE, Adjei AA. Non-small cell lung cancer: epidemiology, risk factors, treatment, and survivorship. Mayo Clin Proc. 2008;83(5):584-594.

3. Wang Z. Selection of chemotherapy for non-small cell lung cancer is facilitated by new therapeutic strategies. Int J Clin Exp Med. 2014;7(11):3833-3842.

4. Wangari-Talbot J, Hopper-Borge E. Drug resistance mechanisms in non-small cell lung carcinoma. $J$ Can Res Updates. 2013;2(4):265-282.

5. Paul I, Jones JM. Apoptosis block as a barrier to effective therapy in non small cell lung cancer. World J Clin Oncol. 2014;5(4):588-594.

6. Stewart DJ. Tumor and host factors that may limit efficacy of chemotherapy in non-small cell and small cell lung cancer. Crit Rev Oncol Hematol. 2010;75(3):173-234.

7. Mottamal M, Zheng S, Huang TL, Wang G. Histone deacetylase inhibitors in clinical studies as templates for new anticancer agents. Molecules. 2015;20(3):3898-3941.

8. Ansari J, Shackelford RE, El-Osta H. Epigenetics in non-small cell lung cancer: from basics to therapeutics. Transl Lung Cancer Res. 2016;5(2):155-171.

9. Bolden JE, Shi W, Jankowski K, et al. HDAC inhibitors induce tumorcell-selective pro-apoptotic transcriptional responses. Cell Death Dis. 2013;4:e519.

10. Sharma S, Kelly TK, Jones PA. Epigenetics in cancer. Carcinogenesis. 2010;31(1):27-36.

11. Housman G, Byler S, Heerboth S, et al. Drug resistance in cancer: an overview. Cancers (Basel). 2014;6(3):1769-1792.

12. Hu E, Dul E, Sung CM, et al. Identification of novel isoform-selective inhibitors within class I histone deacetylases. J Pharmacol Exp Ther. 2003;307(2):720-728.

13. Monneret C. Histone deacetylase inhibitors. Eur J Med Chem. 2005;40(1):1-13.

14. Chang TH, Szabo E. Enhanced growth inhibition by combination differentiation therapy with ligands of peroxisome proliferator-activated receptor-gamma and inhibitors of histone deacetylase in adenocarcinoma of the lung. Clin Cancer Res. 2002;8(4):1206-1212.

15. Zhang X, Wei L, Yang Y, Yu Q. Sodium 4-phenylbutyrate induces apoptosis of human lung carcinoma cells through activating JNK pathway. J Cell Biochem. 2004;93(4):819-829.

16. Belinsky SA, Klinge DM, Stidley CA, et al. Inhibition of DNA methylation and histone deacetylation prevents murine lung cancer. Cancer Res. 2003;63(21):7089-7093.

17. Lyon CM, Klinge DM, Do KC, et al. Rosiglitazone prevents the progression of preinvasive lung cancer in a murine model. Carcinogenesis. 2009;30(12):2095-2099.

18. Al-Keilani MS, Al-Sawalha NA. Potential of phenylbutyrate as adjuvant chemotherapy: An overview of cellular and molecular anticancer mechanisms. Chem Res Toxicol. 2017;30(10):1767-1777. 
19. Martinez-Marignac V, Shawi M, Pinedo-Carpio E, et al. Pharmacological targeting of eIF4E in primary CLL lymphocytes. Blood Cancer J. 2013;3:e146.

20. Caulin AF, Maley CC. Peto's Paradox: evolution's prescription for cancer prevention. Trends Ecol Evol. 2011;26(4):175-182.

21. Kim MG, Pak JH, Choi WH, Park JY, Nam JH, Kim JH. The relationship between cisplatin resistance and histone deacetylase isoform overexpression in epithelial ovarian cancer cell lines. J Gynecol Oncol. 2012;23(3):182-189.

22. Fischer C, Leithner K, Wohlkoenig C, et al. Panobinostat reduces hypoxia-induced cisplatin resistance of non-small cell lung carcinoma cells via HIF-1 $\alpha$ destabilization. Mol Cancer. 2015;14:4.

23. To KK, Tong WS, Fu LW. Reversal of platinum drug resistance by the histone deacetylase inhibitor belinostat. Lung Cancer. 2017;103:58-65.

24. Minamiya Y, Ono T, Saito H, et al. Expression of histone deacetylase 1 correlates with a poor prognosis in patients with adenocarcinoma of the lung. Lung Cancer. 2011;74(2):300-304.

25. Osada H, Tatematsu Y, Saito H, Yatabe Y, Mitsudomi T, Takahashi T. Reduced expression of class II histone deacetylase genes is associated with poor prognosis in lung cancer patients. Int J Cancer. 2004;112(1):26-32.

26. Zhang QC, Jiang SJ, Zhang S, Ma XB. Histone deacetylase inhibitor trichostatin A enhances anti-tumor effects of docetaxel or erlotinib in A549 cell line. Asian Pac J Cancer Prev. 2012;13(7):3471-3476.
27. Kopp F, Wagner E, Roidl A. The proto-oncogene KRAS is targeted by miR-200c. Oncotarget. 2014;5(1):185-195.

28. Van Schaeybroeck S, Kyula J, Kelly DM, et al. Chemotherapy-induced epidermal growth factor receptor activation determines response to combined gefitinib/chemotherapy treatment in non-small cell lung cancer cells. Mol Cancer Ther. 2006;5(5):1154-1165.

29. Greve G, Schiffmann I, Pfeifer D, Pantic M, Schuler J, Lubbert M. The pan-HDAC inhibitor panobinostat acts as a sensitizer for erlotinib activity in EGFR-mutated and -wildtype non-small cell lung cancer cells. BMC Cancer. 2015;15:947.

30. Zhang W, Peyton M, Xie Y, et al. Histone deacetylase inhibitor romidepsin enhances anti-tumor effect of erlotinib in non-small cell lung cancer (NSCLC) cell lines. J Thorac Oncol. 2009;4:161.

31. Kurtze I, Sonnemann J, Beck JF. KRAS-mutated non-small cell lung cancer cells are responsive to either co-treatment with erlotinib or gefitinib and histone deacetylase inhibitors or single treatment with lapatinib. Oncol Rep. 2011;25(4):1021-1029.

32. Sekhavat A, Sun JM, Davie JR. Competitive inhibition of histone deacetylase activity by trichostatin A and butyrate. Biochem Cell Biol. 2007;85(6):751-758.

33. Jin X, Wu N, Dai J, Li Q, Xiao X. TXNIP mediates the differential responses of A549 cells to sodium butyrate and sodium 4-phenylbutyrate treatment. Cancer Med. 2017;6(2):424-438.
Clinical Pharmacology: Advances and Applications

\section{Publish your work in this journal}

Clinical Pharmacology: Advances and Applications is an international, peer-reviewed, open access journal publishing original research, reports, reviews and commentaries on all areas of drug experience in humans. The manuscript management system is completely online and includes a very quick and fair peer-review system, which is all easy to use.

\section{Dovepress}

Visit http://www.dovepress.com/testimonials.php to read real quotes from published authors. 